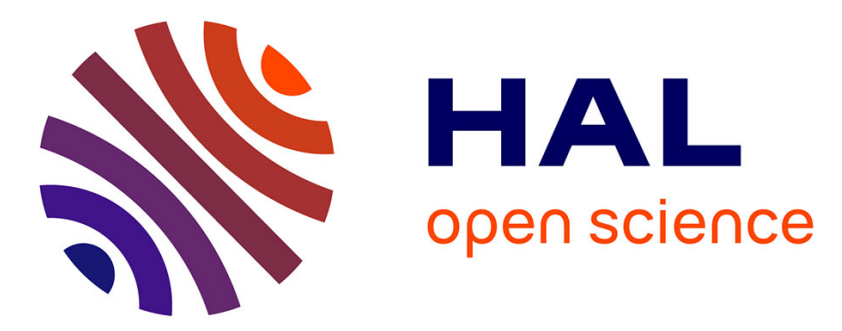

\title{
Consommation de sucre et risque de cancer du sein dans la cohorte NutriNet-Santé
}

Charlotte Debras, Eloi Chazelas, Bernard Srour, Laurent Zelek, Emmanuelle

Kesse-Guyot, Chantal Julia, Nathalie Druesne-Pecollo, Pilar Galan, Serge

Hercberg, Paule Latino-Martel, et al.

\section{To cite this version:}

Charlotte Debras, Eloi Chazelas, Bernard Srour, Laurent Zelek, Emmanuelle Kesse-Guyot, et al.. Consommation de sucre et risque de cancer du sein dans la cohorte NutriNet-Santé. Journées Francophones de Nutrition, Nov 2019, Rennes, France. inserm-02438232

\section{HAL Id: inserm-02438232 https://www.hal.inserm.fr/inserm-02438232}

Submitted on 14 Jan 2020

HAL is a multi-disciplinary open access archive for the deposit and dissemination of scientific research documents, whether they are published or not. The documents may come from teaching and research institutions in France or abroad, or from public or private research centers.
L'archive ouverte pluridisciplinaire HAL, est destinée au dépôt et à la diffusion de documents scientifiques de niveau recherche, publiés ou non, émanant des établissements d'enseignement et de recherche français ou étrangers, des laboratoires publics ou privés. 


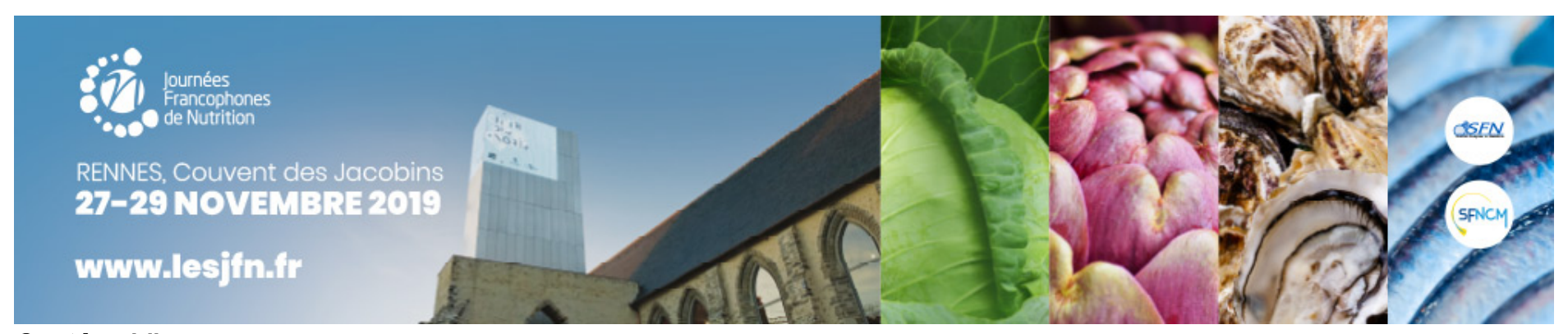

Santé publique

JFN2019/200

\section{Consommation de sucre et risque de cancer du sein dans la cohorte NutriNet-Santé}

Charlotte F. Debras ${ }^{*}$, Eloi Chazelas ${ }^{1}$, Bernard Srour ${ }^{1}$, Laurent Zelek², Emmanuelle Kesse-Guyot ${ }^{1}$, Chantal Julia ${ }^{1,3}$, Nathalie Druesne-Pecollo1, Pilar Galan ${ }^{1}$, Serge Hercberg', ${ }^{1}$, Paule Latino-Martel ${ }^{1}$, Mélanie Deschasaux ${ }^{1}$, Mathilde Touvier $^{1}$

${ }^{1}$ Equipe de Recherche en Epidémiologie Nutritionnelle (Inserm 1153/Inra 1125/Cnam/Université de Paris - Paris 13), ${ }^{2}$ Département d'oncologie, ${ }^{3}$ Département de santé public, Hôpital Avicenne AP-HP, Bobigny, France

Discipline: Epidémiologie

Présentation préférée: Communication orale

Spécifiez votre âge: $<30$ ans

Introduction et but de l'étude: L'impact de la consommation de sucre sur le risque de diabète et de maladies cardiovasculaires est aujourd'hui bien établi. En revanche, le niveau de preuve concernant le rôle joué par les sucres simples sur l'apparition de cancers est très limité, or ils pourraient avoir un effet délétère sur le risque de cancer via différents mécanismes, tels que la prise de poids, mais également l'inflammation, le stress oxydant ou la résistance à l'insuline. Nous avons récemment montré que la consommation de boissons sucrées était positivement associée à l'incidence de cancer, en particulier de cancer du sein, dans la cohorte NutriNet-Santé (Chazelas BMJ 2019). L'objectif était ici d'étendre cette recherche à tous les produits sucrés et d'étudier prospectivement l'association entre la consommation de sucres (sucres simples totaux, sucres ajoutés, et sucres provenant de différentes sources) et le risque de cancer du sein (au global et selon le statut ménopausique) au sein d'une large cohorte d'adultes français.

Matériel et méthodes: Cette étude est basée sur une cohorte prospective. Au total 79742 femmes de plus de 18 ans (âge moyen : $41,0 \pm 14,1)$ de la cohorte NutriNet-Santé (2009-2019) ont été incluses. La consommation de sucre été évaluée à l'aide d'enregistrements alimentaires de 24 heures répétés, conçus pour évaluer les consommations habituelles des participants pour plus de 3500 items alimentaires. Les associations entre la consommation de sucre et le risque de cancers du sein (préménopausique, postménopausique et au global) ont été évaluées à l'aide de modèles de Cox multi-ajustés sur les principaux facteurs de risque connus. Résultats et Analyse statistique : La consommation de sucre était significativement associée avec un risque plus élevé de cancer du sein ( $\mathrm{n}=783, \mathrm{HR}_{\mathrm{Q} 4 \text { vs. Q1 }}=1,51$; intervalle de confiance à $95 \%: 1,14-2,00 ; \mathrm{P}$ de tendance $\left.=0,0007\right)$, de même pour la consommation de sucres ajoutés ( $\mathrm{n}=783$, $\left.\mathrm{HR}_{\mathrm{Q} 4 \text { vs. Q1 }}=1,47 ; \mathrm{IC}_{95 \%}: 1,12-1,91 ; \mathrm{P}=0,02\right)$. Ces associations étaient plus spécifiquement observées chez les femmes pré-ménopausées ( $\mathrm{P}=0.003$ pour les sucres simples et $\mathrm{P}=0.03$ pour les sucres ajoutés, 324 cas), celles-ci consommant plus de produits sucrées que les femmes post-ménopausées, chez qui les tendances correspondantes n'étaient pas significatives ( $\mathrm{P}=0,06$ pour les sucres simples et 0,3 pour les sucres ajoutés, 459 cas). Concernant les sources alimentaires, les sucres simples des boissons $\left(\mathrm{HR}_{\mathrm{Q} 4 \text { vs. Q1 }}=1.38 ; \mathrm{IC}_{95 \%}: 1,09-1,74 ; \mathrm{P}=0,002\right)$, des produits laitiers $\left(\mathrm{HR}_{\mathrm{Q} 4 \text { vs. Q1 }}=1.32\right.$; $\left.\mathrm{IC}_{95 \%}: 1,05-1,65 ; \mathrm{P}=0,01\right)$, et des desserts lactés sucrés $\left(\mathrm{HR}_{\mathrm{Q} 4 \text { vs. Q1 }}=1.29 ; \mathrm{IC}_{95 \%}: 1,05-1,59 ; \mathrm{P}=0,02\right)$ étaient plus particulièrement associés au risque de cancer du sein.

Conclusion: Cette large étude prospective suggère une association entre consommation accrue de sucre et risque de cancer du sein. Ces résultats doivent être reproduits dans d'autres études prospectives à grande échelle. Ils suggèrent que le sucre et les aliments sucrés, dont la consommation augmente dans les pays occidentaux, pourraient représenter un facteur de risque modifiable pour la prévention primaire du cancer du sein.

Je confirme que ce résumé est approuvé par l'ensemble des auteurs: Oui Je confirme avoir déclaré ci-dessus les conflits d'intérêts (pour les 5 dernières années) de chaque co-auteur de ce travail: Oui

Dans l'éventualité d'une publication dans les revues scientifiques de la SFNCM ou de la SFN, j'accepte que mon résumé soit publié, avec les coordonnées de l'auteur soumettant: Oui

Conflits d'intérêts: Aucun conflit à déclarer 